\title{
$\mathrm{AIP} \mid$ chaos \\ An Interdisciplinary Journal of Nonlinear Science
}

\section{A dynamical systems approach to spiral wave dynamics}

Dwight Barkley and loannis G. Kevrekidis

Citation: Chaos 4, 453 (1994); doi: 10.1063/1.166023

View online: http://dx.doi.org/10.1063/1.166023

View Table of Contents: http://scitation.aip.org/content/aip/journal/chaos/4/3?ver=pdfcov

Published by the AIP Publishing

\section{Articles you may be interested in}

Reversal of spiral waves in an oscillatory system caused by an inhomogeneity

Chaos 23, 033130 (2013); 10.1063/1.4819900

Three-dimensional spiral waves in an excitable reaction system: Initiation and dynamics of scroll rings and scroll ring pairs

Chaos 18, 026102 (2008); 10.1063/1.2896100

Spiral wave dynamics in excitable media with spherical geometries

Chaos 16, 037115 (2006); 10.1063/1.2346237

Complexity in spiral wave dynamicsa)

Chaos 3, 15 (1993); 10.1063/1.165973

On rotating spiral waves in reaction-diffusion systems

AIP Conf. Proc. 256, 499 (1992); 10.1063/1.42419

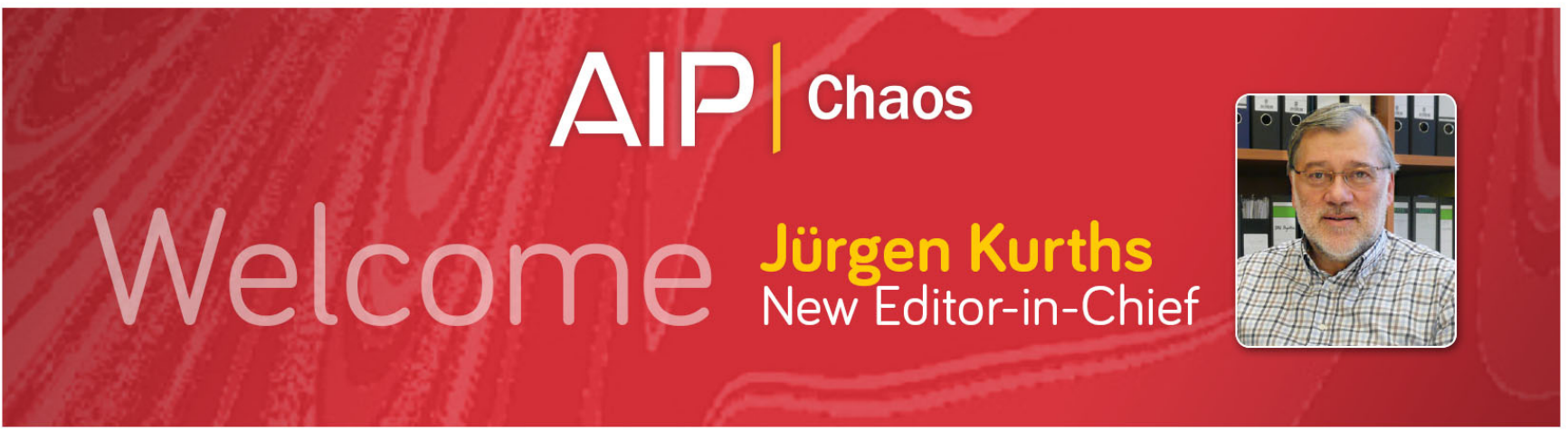




\title{
A dynamical systems approach to spiral wave dynamics
}

\author{
Dwight Barkley ${ }^{\text {a) }}$ \\ Department of Mathematics and Center for Nonlinear Dynamics, University of Texas, Austin, Texas 78712 \\ and Laboratoire de Physique, Ecole Normale Supérieure de Lyon, 46 Allée d'Italie, 69364 Lyon, France
}

loannis $\mathrm{G}$. Kevrekidis

Department of Chemical Engineering, Princeton University, Princeton, New Jersey 08544

(Received 24 March 1994; accepted for publication 12 July 1994)

A simple system of five nonlinear ordinary differential equations is shown to reproduce many dynamical features of spiral waves in two-dimensional excitable media.

\section{INTRODUCTION}

The spiral waves found in excitable media, such as Belousov-Zhabotinsky reagent, often do not rotate rigidly about stationary centers. For many choices of system parameters, spiral waves execute complicated motions as they rotate. This is now well confirmed both in experimental systems free from dust and other imperfections ${ }^{1-3}$ and in fully resolved numerical simulations of homogeneous twodimensional excitable media. ${ }^{1,4-8}$ References $9-11$ are recent reviews of the subject.

We illustrate the situation in Fig. 1 with a two-parameter phase diagram of wave dynamics in the Fitzhugh-Nagumo model of excitable media. ${ }^{10}$ The model and phase diagram are discussed in detail below. Here we wish simply to show some of the variety of spiral dynamics found in excitable media and to emphasize that distinct dynamical states are separated by curves (bifurcation loci) in parameter space.

What is particularly interesting and important about the phase diagram in Fig. 1 is its ubiquity: all two-parameter surveys of spiral dynamics in excitable media, both computational and experimental, have revealed states and transitions similar to those in Fig. 1, organized approximately in the same way. ${ }^{4,10,12,13}$ As yet there is no explanation for the genericity of spiral-wave behavior.

In this paper, we offer a very simple picture of spiral dynamics and take the first steps toward explaining why the behavior of spiral waves in diverse excitable media is typically organized in parameter space qualitatively as shown in Fig. 1. Our approach is to examine a minimal dynamical system (system of ordinary differential equations) which possesses certain key bifurcations and symmetries of spiral waves. The behavior which follows from only such general considerations will necessarily be independent of the details of any particular excitable system. Thus we can readily determine which aspects of spiral dynamics depend on system details and which aspects depend only on generic properties of bifurcations with symmetry. The approach we take abounds in the study of nonlinear dynamical systems. ${ }^{14-16}$ Such a dynamical-systems approach is, however, new to the study of spiral waves in excitable media.

\footnotetext{
${ }^{2}$ Present address: Mathematics Institute, University of Warwick, Coventry CV4 7AL, United Kingdom.
}

\section{FITZHUGH-NAGUMO RESULTS}

In this section we elaborate on the dynamics shown in Fig. 1. The results were obtained by Winfree ${ }^{10}$ in a detailed numerical study of the Fitzhugh-Nagumo (FHN) model of excitable media:

$$
\begin{aligned}
& \frac{\partial u}{\partial t}=\nabla^{2} u+f(u, v)=\nabla^{2} u+\epsilon^{-1}\left(u-u^{3} / 3-v\right), \\
& \frac{\partial v}{\partial t}=g(u, v)=\epsilon(u+\beta-\gamma v) .
\end{aligned}
$$

The fields $u$ and $v$ are functions of two-dimensional space in addition to time. The model parameters are $\beta, \gamma$, and $\epsilon$, where $\beta$ controls the asymmetry between excitation and recovery, and $\epsilon$ controls the spatiotemporal scale separation in the system. Further discussion of excitable media and details of the FHN model can be found elsewhere. ${ }^{9-11}$

In his study, Winfree fixed the parameter $\gamma$ at 0.5 , and conducted numerical simulations of Eqs. (1) as a function of the other two parameters, $\beta$ and $\epsilon$. For all parameter values the system has a spatially homogeneous steady state given by $f(u, v)=g(u, v)=0$. For $\beta \gtrsim 2 / 3$ this state is linearly stable; however, it may be nonlinearly unstable, i.e., large perturbations of certain types may not decay to the homogeneous steady state but instead evolve into propagating patterns. We now describe the various regions shown in Fig 1.

For $\beta$ and $\epsilon$ large (lower left of the figure), no wave propagation is possible: all initial conditions evolve to the homogeneous steady state. Heuristically, in this parameter region, the medium is not sufficiently excitable to support wave propagation. The curve $\partial P$ denotes the boundary of propagation: everywhere above this curve the system can sustain plane waves.

While wave propagation is possible everywhere above the curve $\partial P$, spiral waves only exist above the curve $\partial R$ ("rotor boundary"). Thus there is a small region of parameter space (between the curves $\partial P$ and $\partial R$ ) in which wave propagation is possible but spiral waves do not exist.

The best illustration we know for the behavior in the vicinity of the curves $\partial P$ and $\partial R$ is from the work of Zykov and Mikhailov ${ }^{17}$ reproduced in Fig. 2. Zykov and Mikhailov did not study the FHN model, but a similar model. The behavior under discussion is, nevertheless, generic for spiral 


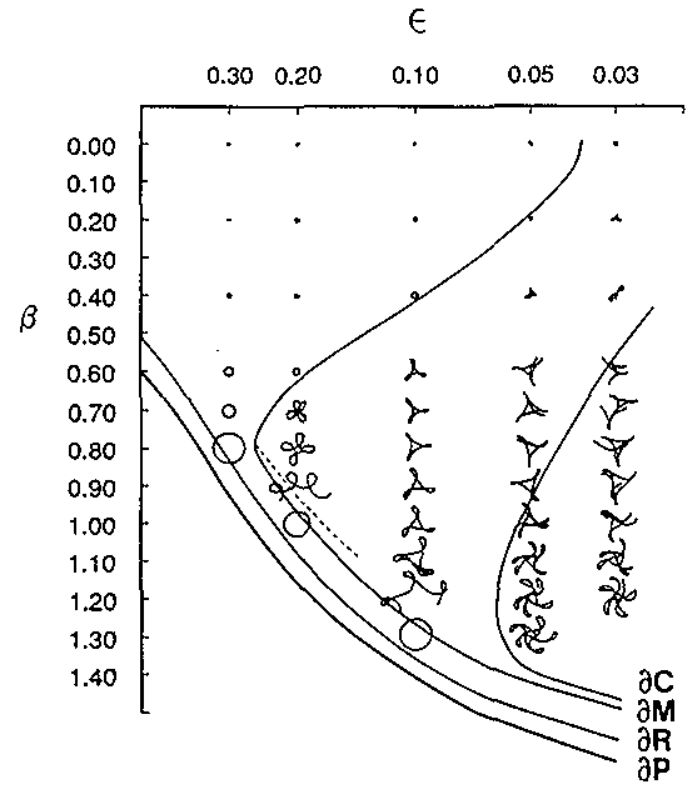

FIG. 1. Phase diagram for spiral-wave dynamics in the FHN model with $\gamma=0.5$. Adapted with permission from Ref. 10 . Curves separating various regions arc labeled as follows. $\partial P$ denotes the limit of propagating solutions. $\partial R$ the rotor boundary: the limit of rotating-wave solutions. $\partial M \mathrm{de}-$ notes the transition to "meandering" waves, which we call modulated waves. $\partial C$ denotes the transition to "complex" states. The dashed curve denotes the locus of modulated-traveling waves. Shown are tip-path plots centered at corresponding parameter points. The circles correspond to rotating waves; "flowers" correspond to various types of modulated and complex states.

waves and the same transitions exist in the model they studied. (See also Refs. 18 and 19 for discussion of the behavior near $\partial R$.)

Figure 2 shows the propagation of an initial broken wave segment for 4 different cases. Consider first Fig. 2(d). The model parameters lie inside the region of spiral waves (corresponding to above $\partial R$ in Fig. 1), and the initial broken segment evolves into a rotating spiral wave. This is also true for Fig. 2(c); here, however, the wave tip rotates with such a large radius that the spiral wave will not fit inside the domain shown. In Fig. 2(b) the tip-path radius has become infinite and the initial segment propagates along a straight line. Case 2 (b) is precisely at the boundary $\partial R$. Figure 2(a) shows a case between $\partial R$ and $\partial P$ : wave propagation is possible, but spiral waves do not exist. In such cases wave tips are said to retract. Note that retracting wave tips propagate along straight lines throughout the region between $\partial R$ and $\partial P$. Also note that at the limit of propagation, $\partial P$, wave segments generally propagate at finite speed.

We now turn to the spiral dynamics shown above $\partial R$ in Fig. 1. To represent the various spiral states, Winfree plots a segment of the path traced out by the tip of the spiral as it evolves in time. Each tip trace is centered on the corresponding parameter point.

The region of periodic states lies above $\partial R$ and to the left of the curve $\partial M$. These periodic states are rotating waves, that is, these spirals rotate rigidly about fixed centers and there is a uniformly rotating frame of reference in which
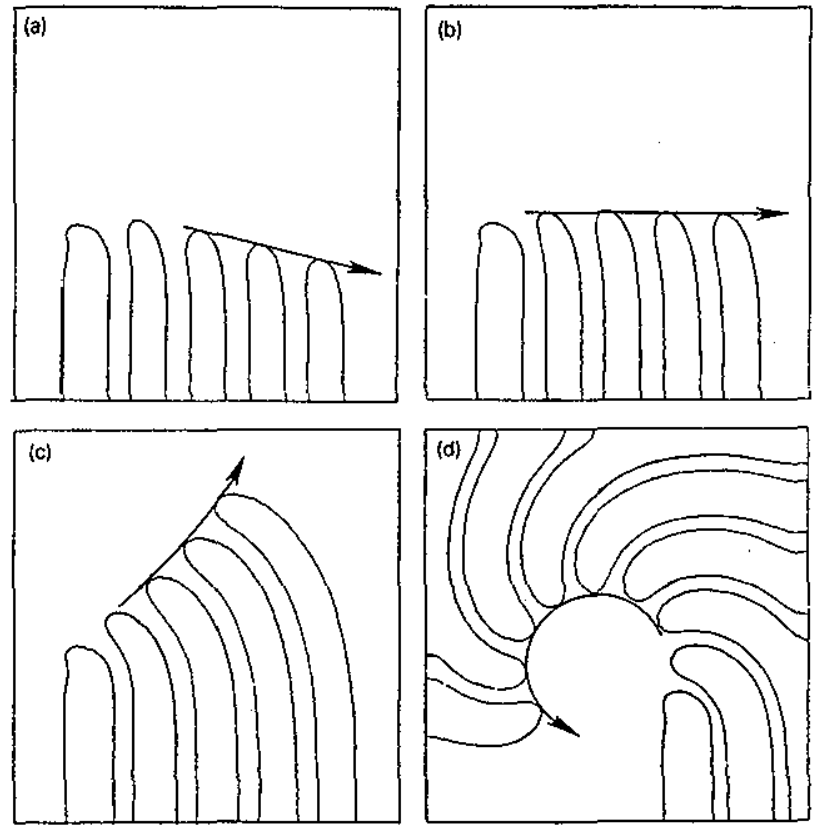

FIG. 2. Illustration of the bchavior of a wave segment for parameters near the rotor boundary of an excitable medium (e.g. near the curve $\partial R$ in Fig. 1). Adapted with permission from Ref. 17. Details can be found there, (a) Parameter values between $\partial P$ and $\partial R$ : the initial segment retracts. (b) $\mathrm{Pa}$ rameter values exactly on the curve $\partial R$. (c), (d) Parameter values inside the region of rotating-wave solutions: initial segments evolve into rotating waves; (c) is closer to the rotor boundary than (d).

they are steady. ${ }^{6,13,16,20}$ Their tip paths form circles. We shall let $\omega_{1}$ denote the rotation frequency of rotating-wave solutions. For $\beta$ near zero, the tip-path radius is quite small. Near the curve $\partial R$ the radius becomes large, and as $\partial R$ is approached, the tip-path radius, spiral period, and spiral wavelength all diverge to infinity. ${ }^{10,17-19}$

The curve $\partial M$ denotes the transition to "meandering." In a numerical linear stability study of a model similar to the FHN, the curve $\partial M$ has been shown to be a single smooth locus of Hopf bifurcations from the rotating spiral waves. ${ }^{13}$ Throughout most of the region between $\partial M$ and $\partial C$, the states are modulated rotating waves (MRW). These are twofrequency quasiperiodic states that appear as periodic in a uniformly rotating frame. ${ }^{6,13,16,20}$ In the context of excitable media, the MRW states are usually referred to as meandering ${ }^{1}$ or as compound rotations. ${ }^{3}$ We shall let $\omega_{2}$ denote the secondary (Hopf) frequency which arises at the Hopf bifurcation.

Figure 3 shows tip paths from simulations of the FHN model in addition to those shown directly in Fig. 1. The tip paths of the MRW form "flower" patterns. The flowers are of two types, those with inward petals [e.g. Figs. 3(c) and 3(d)], and those with outward petals [e.g. Figs. 3(a) and 3(b)].

Separating the two distinct flower types is a curve in parameter space (dashed curve in Fig. 1) on which the flower size is infinite. Winfree shows only a portion of this curve in Fig. 1; it continues to larger $\beta$ and smaller $\epsilon$. Figure 3(e) shows a tip-path segment for an "infinite flower." These 


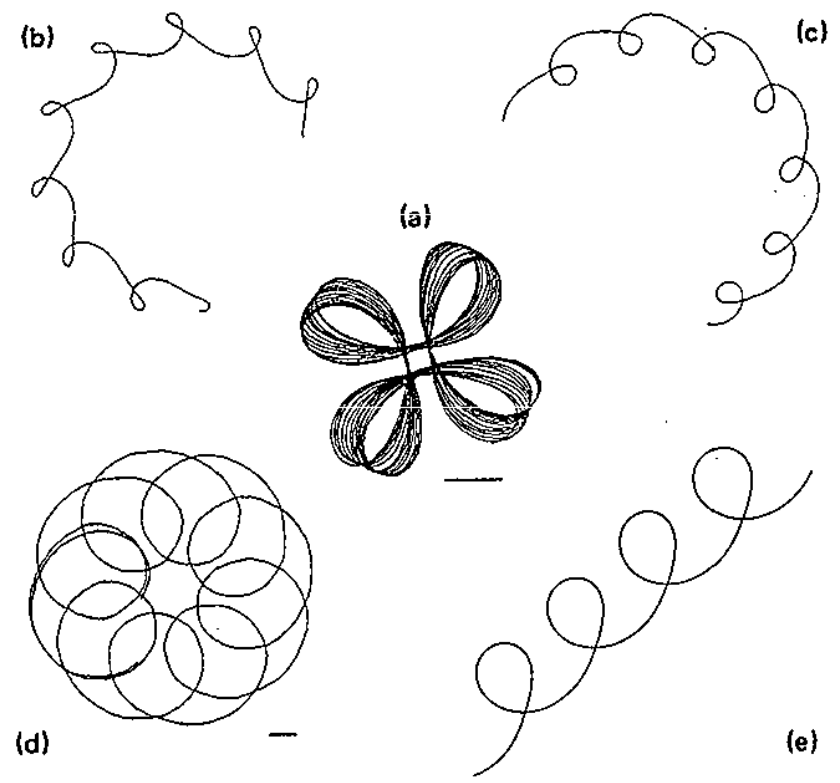

FIG. 3. Spiral tip paths for the FHN model at scveral points of the diagram in Fig. 1. Adapted from Ref. 10 with permission. (a) and (b) are modulatedrotating-wave (MRW) states with outward petals lying above the dashed curve in Fig. 1. (c) and (d) are MRW states with inward petals lying below the dashed curve in Fig. 1. (e) is a modulated-traveling-wave (MTW) state lying on the dashed curve in Fig. 1. Parameter values are as in Fig. 1 with (a) $\epsilon=0.20, \beta=0.770$, (b) $\epsilon=0.11, \beta=1.200$, (c) $\epsilon=0.12, \beta=1.200$, (d) $\epsilon=0.20, \beta=0.955$, (c) $\epsilon=0.20, \beta=0.937$.

states are modulated traveling waves (MTW); they are periodic in a uniformly translating reference frame. The value of $\beta$ for the MTW state in Fig. 3(e) lies between that for 3(a) and 3(d) [Figs. 3(a), 3(d), and 3(e) are all for $\epsilon=0.2$ ]. Figures $3(\mathrm{~b})$ and $3(\mathrm{c})$ illustrate large flowers lying near the MTW locus.

The locus of MTW states emerges from the locus of Hopf bifurcations, $\partial M$, at the point where the bifurcating frequency and the rotational frequency are equal. This resonant Hopf bifurcation will play the central role in the analysis which follows. The traveling speed for the MTW states goes to zero as the distance from the Hopf curve goes to zero. Winfree sketches a "corner" in the Hopf locus $\partial M$ at the point of resonance (Zykov ${ }^{4}$ also draws nonsmooth behavior in the curve $\partial M$ at this point). Accurate stability computations ${ }^{13,21}$ using a model similar to the FHN model, ${ }^{22}$ show that the curve $\partial M$ is, in fact, smooth. It is simply difficult to obtain accurate results by direct simulation in this region.

The final region shown in the FHN phase diagram is that to the right of $\partial C$. Winfree calls the states here "hypermeandering" or "complex." While these states are possibly chaotic, the dynamics in this region are uncharacterized as yet except to the extent that they are more complicated than two-frequency quasiperiodic.

\section{ODE MODEL}

The ordinary-differential-equation (ODE) model we consider is motivated by a numerical bifurcation analysis of rotating spiral waves. In particular, the model is based on an analysis of the resonant Hopf bifurcation from rotating spiral waves in which the Hopf frequency is equal to the rotation frequency of the spiral wave. This analysis is presented in separate publications. ${ }^{13,21}$ Our emphasis here shall be primarily on the global behavior of the model equations, and in particular, on the extent to which the ODE model reproduces the phase diagram shown in Fig. 1 .

We recount important features of the bifurcation analysis and how we are led to the model we consider. The analysis was carried out on a partial-differential-equation (PDE) model of the FHN model type. 22 The following was found: Everywhere on the Hopf locus (analogous to $\partial M$ in Fig. 1), rotating spiral waves have five eigenvalues on the imaginary axis (neutral eigenvalues); all other eigenvalues have negative real part. Of the five eigenvalues, there is a complexconjugate pair associated with the Hopf bifurcation to modulated waves, $\lambda= \pm i \omega_{2}$, where $\omega_{2}$ is the secondary (Hopf) frequency arising at the bifurcation. The remaining three eigenvalues are associated with symmetries of the plane: there is a real eigenvalue due to rotational symmetry, $\lambda=0$, and a complex pair due to translational symmetry, $\lambda= \pm i \omega_{1}$, where $\omega_{1}$ is the primary rotation frequency of the spiral wave. ${ }^{23}$

By simultaneously adjusting two parameters, it is possible to find a codimension-two point in parameter space at which the bifurcating frequency at the Hopf bifurcation equals the primary rotation frequency of the spiral: $\omega_{1}=\omega_{2}$. At this point the complex pair of Hopf eigenvalues comes into coincidence with the complex pair of eigenvalues due to translational symmetry. In addition, as the two parameters are adjusted to move toward the codimension-two point, the complex pair of eigenvectors associated with the Hopf bifurcation align themselves with the complex pair of eigenvectors associated with translational invariance. At the codimension-two point the two pairs of eigenvectors coincide and we find a single complex pair of eigenvectors plus a complex pair of generalized eigenvector.

Such a codimension-two resonant Hopf point exists in Fig. 1: it is at the apex of the Hopf locus $\partial M$ where the curve of modulated-traveling wave solutions emanates from the Hopf locus. In the vicinity of such a codimension-two point there are stable rotating waves, modulated rotating waves of both types (inward and outward petals), and modulated traveling waves.

The low-dimensional ODE model that we consider has been chosen to capture the resonant Hopf bifurcation as simply as possible. That is, we study a simple, weakly nonlinear system of ODEs which (i) are invariant under a representation of distance-preserving transformations of the plane (rotations, reflection, and translations), and which (ii) have rotating-wave solutions which undergo a Hopf bifurcation. By adjusting two parameters of the model, it is possible to bring the Hopf eigenvalues into coincidence with the neutral eigenvalues due to translational symmetry. At such a point the model has a resonant Hopf bifurcation of the same type as the PDE models.

The ODE model is of the following form: 


$$
\begin{aligned}
& \dot{p}=v, \\
& \dot{v}=v \cdot\left\{f\left(|v|^{2}, w^{2}\right)+i w \cdot h\left(|v|^{2}, w^{2}\right)\right\}, \\
& \dot{w}=w \cdot g\left(|v|^{2}, w^{2}\right),
\end{aligned}
$$

where $p$ and $v$ are complex and $w$ is real. $f, g$, and $h$ are real valued functions specified below. The model system is of real dimension 5 because there are five neutral eigenvalues along the Hopf locus in the PDE system.

For any choice of $f, g$, and $h$, these equations are invariant under the following representations of rotations through angle $\gamma, R_{\gamma}$, reffections, $\kappa$, and translations by $\alpha+i \beta, T_{\alpha \beta}$ :

$$
\begin{aligned}
R_{\gamma}\left(\begin{array}{c}
p \\
v \\
w
\end{array}\right) & =\left(\begin{array}{c}
e^{i \gamma} p \\
e^{i \gamma} v \\
w
\end{array}\right), \\
\kappa\left(\begin{array}{c}
p \\
v \\
w
\end{array}\right) & =\left(\begin{array}{c}
p^{*} \\
v^{*} \\
-w
\end{array}\right), \\
T_{\alpha \beta}\left(\begin{array}{c}
p \\
v \\
w
\end{array}\right) & =\left(\begin{array}{c}
p+\alpha+i \beta \\
v \\
w
\end{array}\right),
\end{aligned}
$$

where * denotes complex conjugation. Thus the ODE model equations are invariant under a representation of distancepreserving transformations of the plane.

Letting $p=x+i y$ and $v=s e^{i \phi}$, with $s \geqslant 0$, the equations become:

$$
\begin{aligned}
& \dot{x}=s \cos \phi, \quad \dot{y}=s \sin \phi, \quad \dot{\phi}=w \cdot h\left(s^{2}, w^{2}\right), \\
& \dot{s}=s \cdot f\left(s^{2}, w^{2}\right), \quad \dot{w}=w \cdot g\left(s^{2}, w^{2}\right) .
\end{aligned}
$$

The lowest-order expansions for $f, g$, and $h$ which we found to give the desired codimension-two bifurcation, and which yield bounded trajectories for the model, are:

$$
\begin{aligned}
& f\left(s^{2}, w^{2}\right)=\alpha_{0}+\alpha_{1} s^{2}+\alpha_{2} w^{2}-s^{4}, \\
& g\left(s^{2}, w^{2}\right)=-1+\beta_{1} s^{2}-w^{2}, \\
& h\left(s^{2}, w^{2}\right)=\gamma_{0} .
\end{aligned}
$$

Taking into account possible rescalings of $v, w$, and time, three coefficients have been set to unit magnitude. This then is our ODE model: Eqs. (2) and (7) or equivalently Eqs. (6) and (7).

Before turning to the analysis of this model we shall make some further observations and simplifications. As the notation suggests, $p=x+i y$ is thought of as the position of the spiral tip. Then $v$ is its linear velocity ( $s$ is the tip speed), and $\dot{\phi}=\gamma_{0} w$ the instantaneous rotational frequency. The fact that $p$ does not enter the right-hand side of the ODE system is synonymous with the fact that the dynamics of a spiral are independent of its position. While we shall appeal to this heuristic interpretation of the equations, we stress that the model is based on a detailed numerical study of reactiondiffusion PDEs.

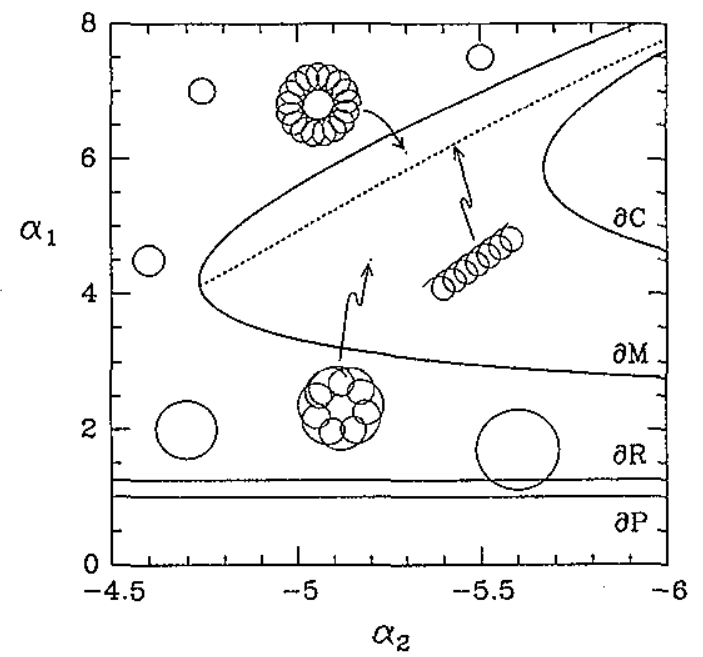

FIG. 4. Phase diagram for the ODE model. Parameters are $\alpha_{0}=-1 / 4, \beta_{1}$, $\beta_{1}=1$, and $\gamma_{0}=5.6 . \partial P$ denotes the locus of saddle-node bifurcations: the limit of propagating solutions. $\partial R$ denotes the locus of drift-pitchfork bifurcations: the limit of rotating-wave solutions. $\partial M$ denotes the locus of Hopf bifurcations from rotating waves: part of the boundary of modulated rotating waves (MRW). $\partial C$ denotes the locus of heteroclinic connections: also part of the boundary of modulated waves. The dashed curve denotes the locus of modulated-traveling waves (MTW). Shown are "tip-path" plots, i.e., plots of $(x, y)$, at various parameter points. The circles correspond to rotating waves. The radii of the circles diverge as $\partial R$ is approached. Two modulatedrotating wave states and a modulated-traveling-wave state are shown.

The $(s, w)$ subsystem in Eqs. (6) decouples from the other three equations and $\phi(t), x(t)$, and $y(t)$ can be found by quadrature once the last two equations are solved. Thus for much of the analysis, we need only consider the dynamics of this subsystem. It is useful to define variables $\xi$ and $\zeta$ by: $\xi=s^{2}$ and $\zeta=w^{2}$. This yields the two-variable system:

$$
\begin{aligned}
& \dot{\xi}=2 \xi f(\xi, \zeta), \\
& \dot{\zeta}=2 \zeta g(\xi, \zeta)
\end{aligned}
$$

We shall refer to this as the reduced system and Eqs. (6) as the full system.

The final simplification which we make it to set $\alpha_{0}=-1 / 4$ and $\beta_{1}=1$ for the remainder of the paper. These parameter values give typical behavior and yet simplify later expressions. With these,

$$
\begin{aligned}
& f(\xi, \zeta)=-\frac{1}{4}+\alpha_{1} \xi+\alpha_{2} \zeta-\xi^{2}, \\
& g(\xi, \zeta)=\xi-\zeta-1 .
\end{aligned}
$$

\section{RESULTS}

In Fig. 4 we show a phase diagram for the ODE model as a function of the two parameters $\alpha_{1}$ and $\alpha_{2}$ with fixed $\gamma_{0}$. We have plotted the diagram so as to emphasize its similarity to the FHN phase diagram in Fig. 1. In the model phase diagram there exist rotating waves, modulated rotating waves of both types (inward and outward petals), and modulated traveling waves. In addition, we find a curve in param- 


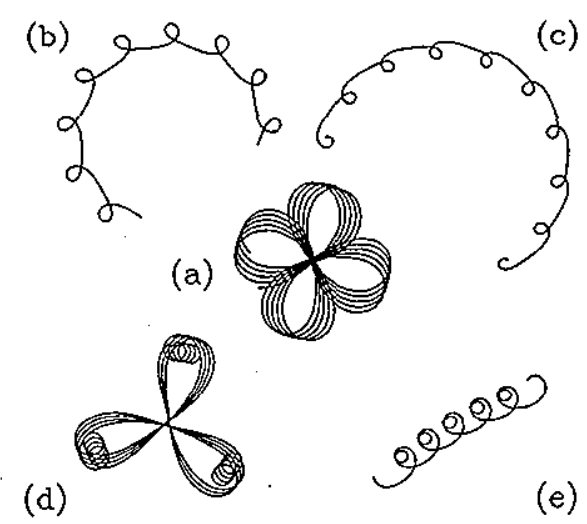

FIG. 5. Plots of $(x, y)$ for the ODE model at various parameter values. (a)-(c) illustrate plots which resemble tip-path plots for spiral waves in the FHN model, i.e., Fig. 3(d) and (e) illustratc plots which do not resemble known tip-path plots for spiral waves. For all plots: $\alpha_{0}=-1 / 4$ and $\beta_{1}=1.0$. The other parameters valucs are: (a) $\alpha_{1}=5.0, \alpha_{2}=-5.5$, and $\gamma_{0}=3.0$. (b) $\alpha_{1}=6.95, \alpha_{2}=-5.8$, and $\gamma_{0}=3.1$. (c) $\alpha_{1}=6.95, \alpha_{2}=-5.8$, and $\gamma_{0}=3.6$. (d) $\alpha_{1}=6.95, \alpha_{2}=-5.8$, and $\gamma_{0}=5.6$. (c) $\alpha_{1}=5.0, \alpha_{2}=-5.5$, and $\gamma_{0}=8.0$.

eter space along which the radius of the rotating waves diverges to infinity and a second curve which is the existence boundary for traveling solutions.

Figure 5 shows model "tip-path" plots, $(x, y)$, for several choices of $\alpha_{1}, \alpha_{2}$, and $\gamma_{0}$. Figures 5(a) -5 (c) are examples which bear a striking resemblance to spiral tip paths in excitable media (cf. Fig. 3 and also Refs. 1-6, 8, 10, 12, 13). By choosing the three parameters appropriately, we are easily able to reproduce essentially all types of spiral tip paths reported in the literature on homogeneous excitable media (excluding those paths which lie in the complex region). However, it is also easy to generate plots that do not correspond to any known spiral paths. Figures 5(d) and 5(e) show two such cases. These are cases for which the primary rotation frequency is large compared to the secondary rotation frequency. The choice of $\gamma_{0}$ is very important in determining the $(x, y)$ flower patterns as well as the location of the MTW locus (dashed curve) in phase diagram in Fig. 4.

The remainder of this section is devoted to an analysis of the model dynamics. For reference we show in Fig. 6 phase portraits for the $(s, w)$ subspace at several different parameter points in Fig. 4.

\section{A. Trivial steady state}

The state $\xi=\zeta=0$ is a steady state of Eqs. (8) for all parameter values. By our choice of constant terms in $f$ and $g$, this steady state is linearly stable for all values of $\alpha_{1}$ and $\alpha_{2}$. This is evident in all the phase portraits shown in Fig. 6 .

\section{B. Traveling pulses}

Next we consider the steady states of Eqs. (8) for which $\zeta=0$ and $f(\xi, 0)=0$. For the full system, these states have nonzero speed, $s \neq 0$, but zero rotational frequency, $w=0$. They give propagation along straight lines in the $(x, y)$ subspace and correspond to retracting tips in the FHN model. For purposes of discussion, we shall call them traveling pulses.
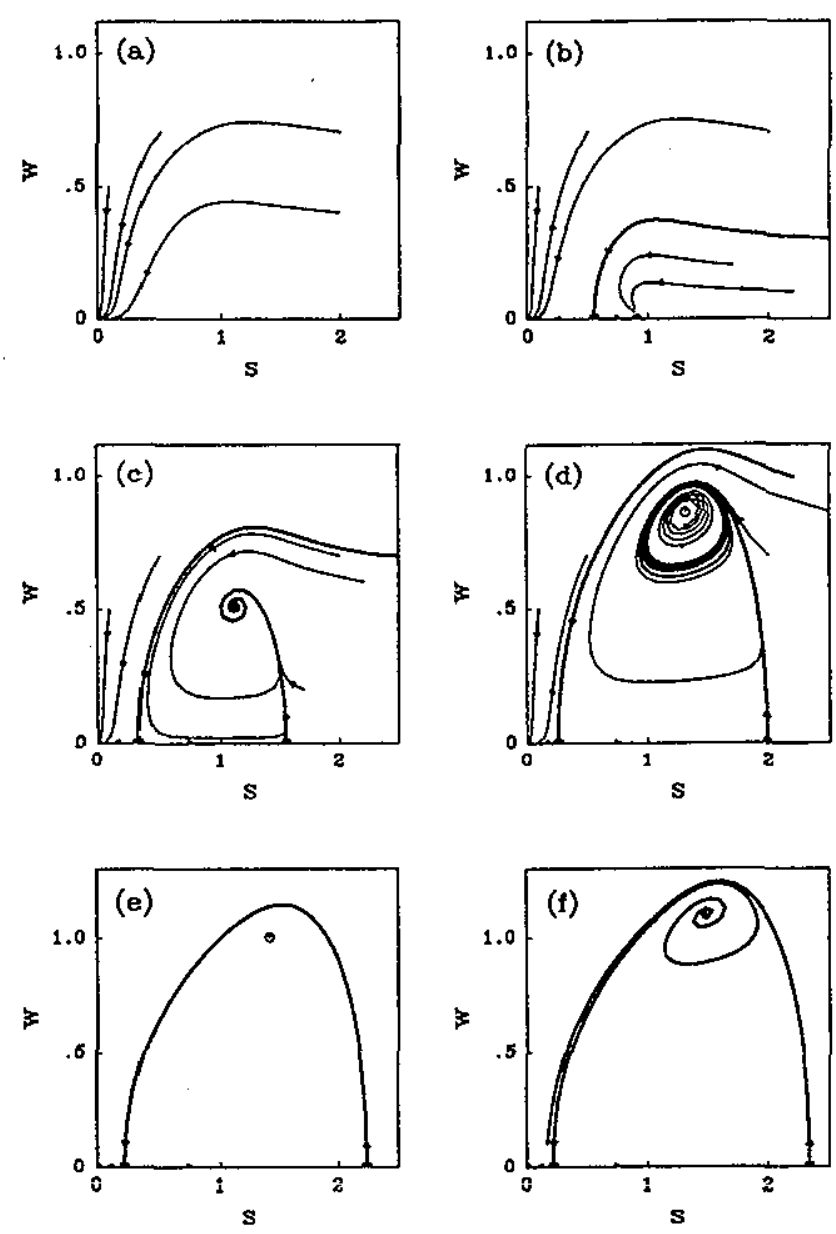

FIG. 6. Phase portraits for the ODE model at scveral parameter points in the diagram in Fig. 4. Shown are the dynamics in the $(s, w)$ subspace. Reflection symmetry implies that the phase portraits arc symmetric about $w=0$ and so only values of $w \geqslant 0$ are shown. (a) Below $\partial P$ : $\alpha_{1}=0.5, \alpha_{2}=-5.0$. The origin in the only asymptotic state and it is stable. (b) Between $\partial P$ and $\partial R: \alpha_{1}=1.125, \alpha_{2}=-5.0$. The saddle and stable node which bifurcate at $\partial P$ are seen. The saddlc lies between the "upper" stable steady statc and the trivial stable steady state at the origin. (c) Above $\partial R$ but below $\partial M: \alpha_{1}=2.5$, $\alpha_{2}=-5.0$. The rotating wave which bifurcates at $\partial R$ is seen as a steady state with nonzero values of $s$ and $w$. (d) Above $\partial M: \alpha_{1}=4.0, \alpha_{2}=-5.0$. The modulated wave which bifurcated from the rotating wave is seen as a stable periodic orbit. The rotating wave is now unstable. (e) On the curve $\partial C$ : $\alpha_{1}=5.040, \alpha_{2}=-5.8$. Heteroclinic connection between the steady states with $w=0$. The period of the periodic orbit in the $(s, w)$ subsystem goes to infinity at the point. (f) Above $\partial C: \alpha_{1}=5.5, \alpha_{2}=-5.8$. After the heteroclinic connection the only stable state is the trivial state at the origin $(0,0)$.

The traveling-pulse states, $\xi_{t p}$, are solutions of $f(\xi, 0)=0$ :

$$
\xi_{t p}^{ \pm}=\frac{\alpha_{1}}{2} \pm \frac{1}{2}\left(\alpha_{1}^{2}-1\right)^{1 / 2}
$$

There are two branches of traveling pulse solutions: an "upper" branch, with "speed" $s^{+}=\sqrt{\xi^{+}}$and a "lower" branch, with "speed" $s^{-}=\sqrt{\xi^{-}}$[Fig. 6(b)]. These branches come together when $\alpha_{1}=1$, and this defines a locus of saddlenode bifurcations. We label the locus of saddle-nodes as $\partial P$ in Fig. 4 to emphasize the similarity with the corresponding curve in Fig. 1. Just as for retracting tips in the FHN system, $\partial P$ in Fig. 4 marks the limit of pulse propa- 
gation: for $\alpha_{1}<1$ there are no traveling pulses and all initial conditions evolve to the trivial state $s=w=0$ [Fig. 6(a)], and at the saddle-node bifurcation, traveling pulses propagate at finite speed: $s_{s n}=\sqrt{\xi_{s n}}=1 / \sqrt{2}$.

A simple linear stability calculation, which we do not reproduce here, shows that the lower branch is unstable for all parameter values we consider. The upper branch is stable for $\alpha_{1}$ between $\partial P$ and $\partial R$ in Fig. 4. At $\partial R$, the upper branch becomes unstable in a pitchfork bifurcation as discussed in the next subsection.

\section{Rotating waves}

Rotating waves are states with constant, nonzero values for $s$ and $w$. They obtain when, in the reduced system, $f(\xi, \zeta)=g(\xi, \zeta)=0$, for positive $\xi$ and $\zeta$. This implies:

$$
\begin{aligned}
& \xi_{1}^{2}-\left(\alpha_{1}+\alpha_{2}\right) \xi_{1}+\alpha_{2}+\frac{1}{4}=0, \\
& \zeta_{1}=\xi_{1}-1 .
\end{aligned}
$$

Only the larger root of Eq. (11) gives a state with positive $\xi$ and $\zeta$ :

$$
\begin{aligned}
& \xi_{1}=\frac{1}{2}\left\{\alpha_{1}+\alpha_{2}+\sqrt{\left(\alpha_{1}+\alpha_{2}\right)^{2}-4 \alpha_{2}-1}\right\}, \\
& \zeta_{1}=\xi_{1}-1 .
\end{aligned}
$$

We define $\omega_{1}$ to be the rotating-wave frequency: $\omega_{1} \equiv \dot{\phi}=\gamma_{0} \cdot w_{1}= \pm \gamma_{0} \sqrt{\zeta_{1}}$. Due to reflectional symmetry, $\kappa$, rotating waves must come in counter-rotating pairs. Those with $\omega_{1}>0$ rotate in the opposite sense from those with $\omega_{1}<0$, and the reflection operator, $\kappa$, takes each of these waves into the other.

Integrating the $\dot{x}$-equation in Eqs. (6) for a rotating wave solution, one obtains:

$$
\begin{aligned}
x(t) & =x_{0}+\left(s_{1} / \omega_{1}\right) \sin \left(\omega_{1} t\right) \\
& =x_{0}+R \sin \left(\omega_{1} t\right)
\end{aligned}
$$

and similarly for $y$, where $R \equiv s_{1} / \omega_{1}$ is the radius of the rotating wave in the $(x, y)$ subspace: $|R|=\gamma_{0}^{-1} \sqrt{\xi_{1} / \zeta_{1}}$.

The rotating waves are born at $\alpha_{1}=5 / 4$, when the upper branch of travel pulses undergoes a pitchfork bifurcation. This bifurcation locus is labeled $\partial R$ in Fig. 4. Figure 6(c) shows a phase portrait after the pitchfork bifurcation to rotating waves. As the locus of pitchfork bifurcations is approached, the rotational frequency, $\omega_{1}$, goes to zero, and the radius, $|R|$ diverges to infinity. Thus the boundary of rotating waves in the ODE model has the same character as the rotor boundary in the FHN model. However, the rotor boundary in the FHN model is not well understood from the point of view of bifurcation theory, so we cannot make detailed comparisons with the ODE model at this time.

\section{Modulated waves}

Modulated waves in the model system correspond to time-periodic behavior for $s(t)$ and $w(t)$. The bifurcation from rotating to modulated waves is simply a Hopf bifurcation, in the reduced system, from a steady state $\left(\xi_{1}, \zeta_{1}\right)$ to a periodic orbit. An expression for the locus of Hopf bifurcations in the model is easily obtained as follows. The stability matrix for Eqs. (8) is:

$$
2\left[\begin{array}{ll}
\xi f_{\xi}(\xi, \zeta) & \xi f_{\zeta}(\xi, \zeta) \\
\zeta g_{\xi}(\xi, \zeta) & \zeta g_{\zeta}(\xi, \zeta)
\end{array}\right],
$$

where subscripts denote differentiation and for now we drop the subscripts on $\xi$ and $\zeta$.

A necessary condition for a Hopf bifurcation is that the trace of the stability matrix is zero:

$$
\xi f_{\xi}(\xi, \zeta)+\zeta g_{\zeta}(\xi, \zeta)=0
$$

This gives

$$
2 \xi^{2}-\alpha_{1} \xi+\zeta=0 .
$$

This condition, together with the two expressions for the rotating waves, Eqs. (13) and (14), defines the Hopf locus in parameter space. [The determinant of (15) is always of the correct sign.] After a little algebra one obtains the following expression for the Hopf locus:

$$
\begin{aligned}
& \xi_{H}=\frac{1}{4}\left\{\left(\alpha_{1}-1\right)+\sqrt{\alpha_{1}^{2}-2 \alpha_{1}+9}\right\}, \\
& \alpha_{2 H}=\frac{3-2\left(\alpha_{1}+1\right) \xi_{H}}{4\left(\xi_{H}-1\right)} .
\end{aligned}
$$

The first equation gives $\xi_{H}$, the value of $\xi$ at the Hopf bifurcation, in terms of $\alpha_{1}$. The second then gives $\alpha_{2}$ for the Hopf bifurcation in terms of $\xi_{H}$ and $\alpha_{1}$. This Hopf locus is plotted in Fig. 4 and labeled $\partial M$.

The MTW locus emerges from the Hopf locus at the codimension-two point where the Hopf frequency equals the rotation frequency. This point is easily found. We define $\omega_{2}$ to be the Hopf frequency, that is $\omega_{2}=\sqrt{D e t}$, where Det is the determinant of the linearization (15) at the bifurcation. Then

$$
\begin{aligned}
\omega_{2}^{2} & =4 \xi \zeta\left(f_{\xi} g_{\zeta}-f_{\zeta} g_{\xi}\right) \\
& =4 \xi \zeta\left(2 \xi-\alpha_{1}-\alpha_{2}\right),
\end{aligned}
$$

this can be simplified using Eq. (16) to give:

$$
\omega_{2}^{2}=4 \zeta\left(1-\left(\alpha_{2 H}+1\right) \xi_{H}\right) .
$$

Thus the frequency ratio at any point along the Hopf locus is:

$$
\left|\omega_{2} / \omega_{1}\right|=\frac{2}{\gamma_{0}} \sqrt{1-\left(\alpha_{2 H}+1\right) \xi_{H}} .
$$

The resonant Hopf point is where $\left|\omega_{2} / \omega_{1}\right|=1$ on the Hopf locus.

In addition to the dependence on parameters $\alpha_{1}$ and $\alpha_{2}$, the frequency ratio $\left|\omega_{2} / \omega_{1}\right|$ depends on the parameter $\gamma_{0}$. Hence the codimension-two point, and also the locus of MTW states which emerges from this point, depend on $\gamma_{0}$. The other bifurcation loci $\partial P, \partial R, \partial M$, and $\partial C$ in Fig. 4 are all independent of $\gamma_{0}$. Thus, by varying $\gamma_{0}$, it is possible to locate the codimension-two point anywhere on the Hopf curve $\partial M$, or to eliminate this point from the phase diagram entirely. We have chosen $\gamma_{0}$ in Fig. 4 such that the codimension-two point is at the apex of the Hopf curve, so that the model phase diagram most closely resembles the spiral phase diagram in Fig. 1. The location of the 
codimension-two point and the curve of MTW states are arbitrary in the model system. We return to this in Sec. V.

We do not have closed-form solutions for the modulated waves which bifurcate from the rotating waves at $\partial M$. For the modulated waves we resort to numerical integration of Eqs. (6). The $(x, y)$ plots of modulated waves shown in Figs. 4 and 5 have been obtained numerically. The locus of MTW states (dashed curve in Fig. 4) has also been obtained numerically by searching for parameter values which give MTW states.

From our simulations, we have found that the locus of Hopf bifurcations in Fig. 4 is everywhere supercritical, i.e., the various flower patterns grow continuously from circles. Likewise, we have found that the translation speed of the MTW states goes to zero at the Hopf locus and that the translation speed scales as the square-root of the parameter distance from the Hopf locus as an analysis of the codimension-two point predicts. ${ }^{13}$

\section{E. Complex states}

Finally we consider the possibility of complex states in the ODE model. Because there are only two nontrivial variables, $s$ and $w$, in Eqs. (6), the model cannot exhibit dynamics more complicated than modulated waves (quasiperiodic states). Thus, to obtain complex states it is necessary to add additional variables to the model. While we shall not examine the effect of additional variables in any detail here, we point out one important effect they could have.

The model possesses heterociinic orbits. These are connections between the two traveling pulse states $\xi^{+}$and $\xi^{-}$. Figure 6(e) shows such a connection in phase space. In Fig. 4 we label the loci of such connections by $\partial C$. To the right of the curve $\partial C$ in Fig. 4 the trivial steady state, $s=0$, $w=0$, is the only stable state of the system [Fig. 6(f)]. If any other degrees of freedom were added to the model, then generically this connection would become a tangle and in the vicinity of this tangle there would exist complex dynamics (see e.g. Ref. 14). This interpretation of the complex states in spiral systems in rather tenuous and the complex spiral states might instead arise via some other mechanism.

\section{DISCUSSION AND CONCLUSION}

We have shown that it is possible to capture much of the spiral-wave dynamics in excitable media with a very simple system of ordinary differential equations. In so doing, we have demonstrated a new method for investigating and understanding spiral dynamics which is distinct from, but complements other approaches such as the kinematical approach, e.g. Refs. 17 and 24 , the free-boundary approach, e.g. Refs. 18, 19 and 25-29, and direct simulations of reaction-diffusion PDEs, e.g., Refs. 1, 4-8, 10, and 22.

In addition to allowing easy study of spiral dynamics, our approach has provided the first steps toward explaining why phase diagrams like that in Fig. 1 are so ubiquitous in spiral systems. The model we have studied is not based on any specific properties of excitable media. The model is instead based on a particular codimension-two bifurcation: a Hopf bifurcation from rotating waves in which Hopf eigen- modes interact with symmetries of the plane. Thus, the dynamics exhibited by our ODE model are entirely independent of the details of any particular excitable medium. In this sense, the model applies to all excitable media (and, in fact, to any system having such a resonant Hopf bifurcation).

What remains, is the important step of explaining why spiral waves in excitable media generically exhibit the kind of Hopf bifurcation we have considered. Why, for example, are the primary and secondary frequencies for spiral waves always found to be so nearly equal? The ODE model was based on this observation, but does not explain why it is so. This important question must be answered by some other means. Kessler, Levine, and Reynolds have performed stability analyses starting directly from reaction-diffusion equations, e.g., Eqs. (1), in the limit of zero $\epsilon$ and have found unstable modes. ${ }^{30,31}$ Unfortunately, in the limit in which they have been able to obtain results, they do not find unstable complex (Hopf) eigenmodes. Nevertheless, this work is very promising and we hope that ultimately it will be possible to explain why resonant Hopf bifurcations are to be expected for spirals in excitable media.

Another, perhaps related question which our approach has not answered is: Why does the resonant Hopf bifurcation typically occur at the apex of the Hopf locus in spiral systems? In our ODE model, the frequency ratio at the Hopf bifurcation is entirely independent of location on the Hopf locus (see Sec. IV D), and hence, the codimension-two point can be located anywhere on the Hopf locus in the ODE system. In phase diagrams for PDE models, however, the codimension-two point is always near a sharp turn in the Hopf locus (see Fig. 1 and also Refs. 4, 10, 12, and 13). The explanation for this must depend on details of excitable media in some way which we do not currently understand.

Even within the dynamical-systems approach we have taken, the ODE model presented is not complete. For example, we know that including more variables in the model will affect the model dynamics significantly in the vicinity of the curve $\partial C$ in Fig. 4. In addition, high-order expansions for the functions $f, g$, and $h$ in Eqs. (7) might change aspects of the model dynamics. Moreover, at this time, we have not established direct correspondence between parameters of the ODE model and the parameters of any excitable media; however, a comparison of the model phase diagram and the diagram for the FHN model suggests that this can be accomplished.

The ordinary-differential-equation model presented in this work should be viewed as a first step in finding lowdimensional description of spiral wave dynamics. Several important questions still remain open. We believe, however, that soon it will be possible to capture completely the dynamics of spiral waves in an excitable medium with such a low dimensional model. We also hope that our approach can be extended to more complicated situations, such as periodically forced spiral systems ${ }^{32}$ and three-dimensional excitable media. $^{33}$

\section{ACKNOWLEDGMENTS}

We have enjoyed discussing issues in this paper with $\mathrm{A}$. T. Winfree, H. Levine, and W. N. Reynolds. We thank A. T. 
Winfree and A. S. Mikhailov for allowing us to reproduce figures from their works. D.B. thanks the NSF for their support though NSF Grant No. DMS 92-06224 and NATO for their support through Grant No. RCD 92-55315. I.G.K acknowledges support from NSF Grant No. ECS 90-23362 and from the David and Lucile Packard Foundation.

${ }^{1}$ W. Jahnke, W. E. Skaggs, and A. T. Winfrec, J. Phys. Chem. 93, 740 (1989).

${ }^{2}$ T. Plesser, S. C. Müller, and B. Hess, J. Phys. Chem. 94, 7501 (1990).

${ }^{3}$ G. S. Skinner and H. L. Swinney, Physica D 48, 1 (1991).

${ }^{4}$ V. S. Zykov, Biofizika 31, 862 (1986)

${ }^{5}$ E. Lugosi, Physica D 40, 331 (1989).

${ }^{6}$ D. Barkley, M. Kness, and L. S. Tuckerman, Phys. Rev. A 42, 2489 (1990).

${ }^{7}$ A. Karma, Phys. Rev. Lett. 65, 2824 (1990).

${ }^{8}$ W. Jahnke and A. T. Winfree, Int. J. Bifurcation Chaos 1, 455 (1991).

${ }^{9}$ Waves and Patterns in Chemical and Biological Media, edited by $\mathrm{H} . \mathrm{L}$. Swinney and V. I. Krinsky, Physica D 49 (1991).

${ }^{10}$ A. T. Winfree, Chaos 1, 303 (1991).

${ }^{11}$ E. Meron, Phys. Rep. 218, 1 (1992).

${ }^{12}$ Z. Nagy-Ungvarai, J. Ungvarai, and S. C. Müller, Chaos 3, 15 (1993).

${ }^{13}$ D. Barkley, Phys. Rev. Lett. 72, 164 (1994).

${ }^{14} \mathrm{~J}$. Guckenheimer and P. Holmes, Nonlinear Oscillations, Dynamical Systems, and Bifurcations of Vector Fields (Springer, New York, 1983).

${ }^{15}$ J. Guckenheimer, Physica D 20, 1 (1986).

${ }^{16}$ M. Golubitsky, I. Stewart, and D. G. Schaeffer, Singularities and Groups in Bifurcation Theory, Vol. $I I$ (Springer, New York, 1988).

${ }^{17}$ A. S. Mikhailov and V. S. Zykov, Physica D 52, 379 (1991).

${ }^{18}$ P. Pelcé and J. Sun, Physica D 48, 353 (1991).
${ }^{19}$ A. Karma, Phys. Rev. Lett. 66, 2274 (1991).

${ }^{20}$ D. A. Rand, Arch. Rat. Mech. Anal. 79, 1 (1982).

${ }^{21}$ D. Barkley, Phys. Rev. Lett. 68, 2090 (1992).

${ }^{22}$ D. Barkley, Physica D 49, 61 (1991).

${ }^{23}$ In two-dimensions, one expects two neutral eigenvalues, associated with the two-parameter family of translations on the planc. The two eigenmodes duc to this translational symmetry become complex when viewed in a frame rotating at the spiral frequency $\omega_{1}$. This can be verified directly from the linear stability operator. ${ }^{21}$ Stability results obtained in the frame rotating at the spiral frequency (where the spiral wave appears as a steady state) correspond directly to Floquet stability results for the spiral wave when viewed in a nonrotating frame (where it appcars as a periodic orbit) e.g., $m=\exp \left(2 \pi \lambda / \omega_{1}\right)$, where $m$ is the Floquet multiplier and $\lambda$ is the eigenvalue in the rotating frame. Hence the complex eigenvalue pair due to translational symmetry corresponds to two Floquet multipliers at unity: $m=\exp \left(2 \pi \lambda / \omega_{1}\right)=\exp \left( \pm i 2 \pi \omega_{1} / \omega_{1}\right)=1$. The effects of translational symmetry appear even in finite domains, because spiral waves in excitable media are almost entirely unaffected by lateral boundarics. ${ }^{1.3}$

${ }^{24}$ E. Meron, Physica D 49, 98 (1991).

${ }^{25}$ J. P. Keener and J. J. Tyson, Physica D 21, 307 (1986).

${ }^{26}$ A. J. Bernoff, Physica D 53, 125 (1991).

${ }^{27}$ A. Karma, Phys. Rev. Lett, 68, 397 (1992).

${ }^{28}$ D. A. Kessler, H. Levinc, and W. N. Reynolds, Phys. Rev. Lett. 68, 401 (1992).

${ }^{29}$ J. P. Keener, SIAM J. Appl. Math. 52, 1370 (1992).

${ }^{30}$ D. A. Kessler, H. Levine, and W. Reynolds, Phys. Rev. A 46, 5264 (1992).

${ }^{31}$ D. A. Kcssler, H. Levine, and W. N. Reynolds, Physica D 70, 115 (1994).

${ }^{32}$ O. Steinbock, V. Zykov, and S. C. Müller, Nature 366, 322 (1994).

${ }^{33}$ A. T. Winfrec, When Time Breaks Down (Princeton U. P., Princeton, NJ, 1987). 